\title{
ANALYSIS OF DIMENSIONAL CHARACTERISTICS OF THE RIBS ON STEEL BARS FOR REINFORCEMENT OF CONCRETE
}

\author{
BARSIC, G[orana]; BUTKOVIC, I[van]; SIMUNOVIC, V[edran] \& KATIC, M[arko]
}

\begin{abstract}
The main condition for the realization of a reinforced concrete construction is a complete coupling between the concrete and steel, used as reinforcement. Using higher quality steel, the force of the reinforcement has been increased and thus the risk of separation of these two materials. To avoid undesirable slip phenomena, great importance is put on the geometrical features of reinforcement steel used, i.e. the main contribution to the total adhesiveness lies in shear adhesiveness of the ribs or profiles on the surface of steel bars. Based on measured geometric features one can calculate a factor called the factor of adhesion or relative rib area. Therefore testing, i.e. concluding about the reliability of the measuring method with respect to the requirements of ISO 15630-1:2002, is the topic of this study. For that purpose measurements were preformed using two methods in order to calculate the factor of adhesion. First method was the optical method, using the instrument RM 302/202 manufactured by ECM Datensysteme $G M B H$. Furthermore measurements were preformed by the use of conventional length measurement equipment that is commonly available in production, sucs as dial indicators and vernier callipers. The obtained measurement results were subjected to statistical analysis as required by ISO 5725-1984 $(E)$, and based on that the conclusions of the accuracy and reliability of measurement methods were made.

Keywords: reinforcing stee, relative rib area, accuracy and reliability of measurement methods
\end{abstract}

\section{INTRODUCTION}

Reinforced concrete as a combination of concrete and reinforcing steel combines good qualities of both materials, the relatively high compressive strength of concrete and high tensile strength of steel. The fact that concrete and reinforcing steel have close values of thermal expansion coefficients, they stretch eaqualiy at the same temperature and do not produce the opposite stress between each other, makes them the most frequent building material throughout the last century all the way to this day. The main condition for the use of reinforced concrete construction is the coupling between the concrete and steel used as reinforcements.

Using more quality steel, the force of the reinforcements have been increased and, the risk of separation of these two materials. To avoid undesirable slip phenomena, great importance is put on the geometrical features of used reinforcement steel. Measurement results of reinforcing steel geometrical features are used to calculate the adhesion factor between concrete and steel, which is called the relative rib area. This information is very important in making a decision on the application of certain type of reinforcing steel, which ensures sufficient shear adhesion between two materials and prevents slippage of reinforcement in concrete.

\section{GEOMETRICAL FEATURES OF STEEL REINFORCEMENT}

Steel products covered by standard HRN 1130:2008 must have a ribbed surface as defined is Section 7.4.2. of HRN EN 10080:2005 standard. Ribbed steel is characterized by the measures, number and arrangement of longitudinal and transverse ribs. The product must have two or more rows of transverse ribs evenly distributed around the volume. Within each row transverse ribs are also evenly distributed. Ribbed steel can have a longitudinal rib as well, figure 1.

Fig. 1. Ribbed steel with and without longitudinal rib

The specific values of ribs can be determined by the relative rib area $f_{R}$ or by combination of the distance between ribs $c$, height of the rib $a$ and transverse rib angle $\cdot$, or by use of both criteria.

The distance between the transverse ribs, rib height and angle are given in Table 1.

\begin{tabular}{|c|c|c|}
\hline Rib height $\boldsymbol{a}$ & $\begin{array}{c}\text { Distance between } \\
\text { ribs } \boldsymbol{c}\end{array}$ & $\begin{array}{c}\text { Transverse rib angle } \\
\boldsymbol{\beta}\end{array}$ \\
\hline $0.03 d-0.15 d$ & $0.4 d-1.2 d$ & $35^{\circ}-75^{\circ}$ \\
\hline
\end{tabular}

Tab. 1. The values of ribs

Reduced size of transverse ribs must engage at least $75 \%$ of the bar volume that is calculated from the nominal diameter. The angle of inclination rib slope $U^{\circ}$ must be $\geq 45^{\circ}$. In the case of longitudinal ribs their height must not exceed $0.15 d$. 


\subsection{Calculation of relative rib area $\boldsymbol{f}_{R}$}

To determine the relative rib area $f_{R}$ as adhesion factors of concrete and reinforcing steel one must use the results of measurements of geometric features already mentioned in this chapter.

The relative rib area is determined using following equation:

$$
f_{R}=\frac{1}{\pi d} \sum_{i=1}^{n} \frac{\frac{1}{m} \sum_{j=1}^{m} F_{R, i, j} \sin \beta_{i, j}}{c_{i}}+\frac{1}{P} \sum_{k=1}^{q} a_{k}^{\prime}
$$

where:

$n \tilde{n}$ is number of rows of transverse ribs around the volume;

$m \tilde{n}$ is number different angles of inclination in a row of transverse ribs;

$q \tilde{n}$ is number of longitudinal ribs of cold twisted bars.

The second augend applies to cold twisted bars only and must be taken into consideration only if it is $30 \%$ greater than the total value of $f_{R}$.

If the special devices are used, master equations for the relative rib area are not used strictly; the simplified equations can be used instead.

An example of such simplified equation is Simpson's equation:

$$
f_{R}=\left(2 a_{1 / 4}+a_{m}+2_{3 / 4}\right)\left(\pi d-\sum e_{i}\right) \frac{1}{6 \pi d c}+\frac{1}{P} q a^{\prime}
$$

In accordance with the requirements of ISO 1130:2008 standard calculated values of relative rib area $f_{R}$ should not be less than from the values given in Table 2 .

\begin{tabular}{|c|c|c|c|c|}
\hline $\begin{array}{c}\text { Nominal } \\
\text { diameter } \\
(\mathbf{m m})\end{array}$ & $\mathbf{5}$ - $\mathbf{6}$ & $\mathbf{5 , 5}-\mathbf{8 . 5}$ & $\mathbf{9 - 1 0 , 5}$ & $\mathbf{\geq 1 1}$ \\
\hline$f_{R}$ & 0,039 & 0,045 & 0,052 & 0,056 \\
\hline
\end{tabular}

Tab. 2. Relative rib area

\section{COMPARISON OF MEASUREMENT METHODS PRECISION}

Statistical analysis includes an estimation of the parameters that are indicators of the accuracy of repeated and reproduced measurements obtained by use of different measurement methods.

When comparing the accuracy of two measurement methods, one of them is convetionally taken as a reference and is appointed A. In comparison to it, measurement precision of another method, marked B, will be compared.

The comparison includes methods for measurement accuracy achieved in terms of repeatability, or repeated measurements and for reproduced measurements.

Based on the results of measurements and their analysis, an estimation of measurement uncertainty was preformed and $E n$ values were calculated.

Owerall analysis and comparison of measurement results of measurements methods is based on international standard ISO 5725-1984(E).

\subsection{Precision of repeated measurements}

As allready mentioned reference method is marked with $\mathrm{A}$ and one that compares it with the $\mathrm{B}$.

If:

$$
\frac{s_{r B}^{2}}{s_{r A}^{2}} \leq \frac{\chi_{(1-\alpha)}^{2}\left(v_{r B}\right)}{v_{r B}}
$$

can not be argued that the precision of repeated measurements using method $\mathrm{A}$ is statistically greater than the precision of the measurement achived with method $\mathrm{B}$, and vice versa.

If:

$$
\frac{s_{r B}^{2}}{s_{r A}^{2}}>\frac{\chi_{(1-\alpha)}^{2}\left(v_{r B}\right)}{v_{r B}}
$$

then it is statistically confirmed that the measurement method B is less accurate than measurement method A, when repeated measurements are mentioned.

Therefore: $\chi_{(1-\alpha)}^{2}\left(v_{r B}\right)-$ factor $\chi^{2}$ distribution for the probability $(1-\alpha)$ and $v_{r B}$ degrees of freedom.

Number of degrees of freedom $v_{r B}$ is determined using following equation:

$$
v_{r B}=p_{B}\left(n_{B}-1\right)
$$

where:

$p_{B}-$ is a number of measurement series of method B;

$n_{B}$ - is a number of repeated mesurements within the mesurements series of method $B$.

It is preferable that the number of rows and number of repeated measurements for both methods are the same.

\subsection{Precision of reproduced measurements}

If the condition holds:

$$
\frac{s_{R B}^{2}-\left[1-\left(\frac{1}{n_{B}}\right)\right] s_{r B}^{2}}{s_{R A}^{2}-\left[1-\left(\frac{1}{n_{B}}\right)\right] s_{r A}^{2}} \leq \frac{\chi_{(1-\alpha)}^{2}\left(v_{L B}\right)}{v_{L B}}
$$

it can be said that the precision of the method B, at a general level, is not significantly smaller than the precision of the method A.

Therefore: $\chi_{(1-\alpha)}^{2}\left(v_{L B}\right)$ - factor $\chi^{2}$ distribution for the probability $(1-\alpha)$ and $v_{L B}$ degrees of freedom.

Number of degrees of freedom $v_{L B}$ is determined using following equation:

$$
v_{L B}=p_{B}-1
$$

where:

$p_{B}$ - is a number of measurement series of method B.

\section{MEASUREMENT OF THE GEOMETRIC CHARACTERISTIC OF REINFORCED STEEL}

Measurements of geometrical characteristics of the reinforcing steel using two methods were preformed, i.e. by use of optical device RM 302/202 manufacturers ECM Datensysteme GMBH (figure 2) and using the 
conventional hand-held measuring instruments (dial incicator and vernier calliper), on four different samples, which are named according to their the size of diameter, 10, 16, 28 and 16-A.

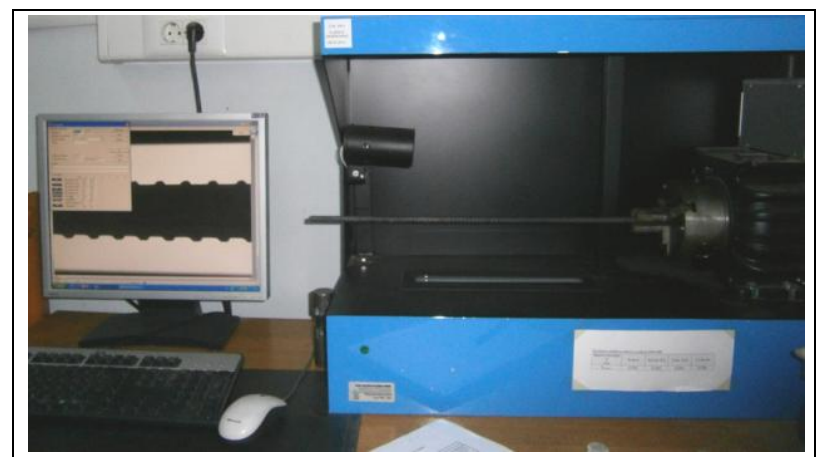

Fig. 2. Optical device RM 302/202

The same transverse ribs were masured on each sample using both measuring methods. Measured characteristics are defined by the standard ISO 156301:2002, as described in the previous chapter, with special accent on characteristics needed to calculate the factor of adhesion as stated by the Simpson's equation. And these characteristics are:

$a_{\mathrm{m}}-$ maximum height of transverse ribs;

$a_{1 / 4}-$ rib height of a quarter of the lenght of transverse ribs;

$a_{3 / 4}-$ rib height of three quarters of the length of transverse ribs;

$c$ - spacing between the transverse ribs;

$e$-spacing between the longitudinal rows of ribs.

The sample marked 16-A has specific geometry, different from other samples. This sample have characteristic longitudinal rib which follows the axis of the rod and is placed on the position where the hight of the transverse ribs is greatest, and its height is half the height of the cross ribs, figure 3 .

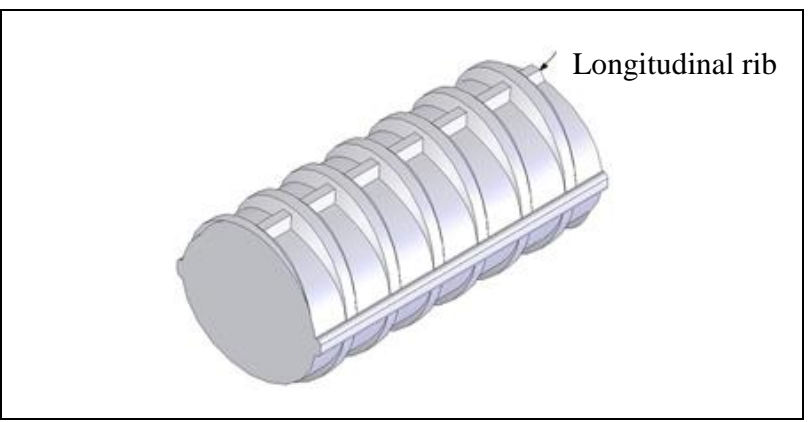

Fig. 3. Longitudinal rib on sample 16-A

Each sample and each characteristic $\left(a_{\mathrm{m}}, a_{1 / 4}, a_{3 / 4}, c\right.$, $e$ ) were measured three times on each side of the sample, i.e. for every row of transverse ribs. The measurement results enabled the calculation of the adhesion factor $f_{R}$ using the Simpson's equation.

For the purposes of statistical analysis measurements were repeated twice. In that way two measurement series with six values for each measured characteristic were obtained. One measurement sequence for one side of the sample, and the second sequence for the other side of the sample.

\subsection{Comparison of the precision of repeated measurements}

As described in chapter 3 , this analysis compares the accuracy of the measurement of $B$ method in relation to the accuracy of the reference A method, for repeated measurements, or measurements realized in terms of repeatability. Results of the precision analysis of repeated measurements for the required characteristics are given in table 3 , where the number of degrees of freedom for B method were $v_{r B}=10$, and $\chi_{(1-\alpha)}^{2}\left(v_{r B}\right)=18,307$ with value $95 \%$.

\begin{tabular}{|c|c|c|c|}
\hline Characteristics & $\frac{\chi_{(\mathbf{1}-\boldsymbol{\alpha})}^{2}\left(\boldsymbol{v}_{\boldsymbol{r} \boldsymbol{B}}\right)}{\left(\boldsymbol{v}_{\boldsymbol{r} \boldsymbol{B}}\right)}$ & & $\frac{\boldsymbol{s}_{\boldsymbol{r} \boldsymbol{B}}^{2}}{\boldsymbol{s}_{\boldsymbol{r} \boldsymbol{A}}^{\mathbf{2}}}$ \\
\hline$a_{\mathrm{m}}$ & 1.83 & $<$ & 1.85 \\
\hline$a_{1 / 4}$ & 1.83 & $>$ & 0.32 \\
\hline$a_{3 / 4}$ & 1.83 & $>$ & 0.43 \\
\hline$c$ & 1.83 & $<$ & 2.89 \\
\hline$e$ & 1.83 & $<$ & 2.81 \\
\hline
\end{tabular}

Tab. 3. Analysis of the precision of repeated measurements

From table 3 it was statistically confirmed that the accuracy of measurements, obtained in terms of repeatability with conventional hand-held measuring instruments, method $\mathrm{B}$, are statistically less significant than precision of optical device RM 302/202-ECM Datensysteme GMBH, method $\mathrm{A}$, in the case of characteristic $a_{\mathrm{m}}, c$ and $e$, while for the characteristics, $a_{1 / 4}$ and $a_{3 / 4}$ we can not claim that.

\subsection{Comparison of accuracy of reproduced measurement}

With this method the precision of measurement methods for measurments achieved in terms of reproducibility were compared. In this analysis, the results of measurements achieved with the optical measuring device RM 302/202 are considered as reference, method $\mathrm{A}$, and with them are compared measurement results achived by conventional hand-held measuring instruments, method $B$. Therefore the estimated standard deviation of reproducibility of method $\mathrm{A}, s_{R A}$ can be marked as $\sigma_{R}$.

The procedure of analysis is described in chapter 3 . In table 4. are given the results of analyzes of the accuracy where the number of degrees of freedom was $v_{L B}=1$, and $\chi_{(1-\alpha)}^{2}\left(v_{L B}\right)=3,843$, with value $95 \%$.

\begin{tabular}{|c|c|c|c|}
\hline \multirow{2}{*}{ Characteristics } & $\frac{\chi_{(\mathbf{1}-\boldsymbol{\alpha})}^{2}\left(\boldsymbol{v}_{\boldsymbol{L} \boldsymbol{B}}\right)}{\left(\boldsymbol{v}_{\boldsymbol{L B}}\right)}$ & & $\frac{\boldsymbol{s}_{\boldsymbol{R} \boldsymbol{B}}^{2}-\left[\mathbf{1}-\left(\frac{\mathbf{1}}{\boldsymbol{n}_{\boldsymbol{B}}}\right)\right] \boldsymbol{s}_{\boldsymbol{r} \boldsymbol{B}}^{2}}{\boldsymbol{s}_{\boldsymbol{R} \boldsymbol{A}}^{2}-\left[\mathbf{1}-\left(\frac{\mathbf{1}}{\boldsymbol{n}_{\boldsymbol{B}}}\right)\right] \boldsymbol{s}_{\boldsymbol{r} \boldsymbol{A}}^{2}}$ \\
\hline$a_{\mathrm{m}}$ & 3.843 & $>$ & 1.374 \\
\hline$a_{1 / 4}$ & 3.843 & $>$ & 2.262 \\
\hline$a_{3 / 4}$ & 3.843 & $>$ & 1.336 \\
\hline$c$ & 3.843 & $>$ & 0.762 \\
\hline$e$ & 3.843 & $>$ & 0.475 \\
\hline
\end{tabular}

Tab. 4. Analysis of the reproduced measurements accuracy

From the results listed in table 4. we can conclude that the general level of precision of the method B is not significantly smaller than the precision of the method A, for all the required characteristics. 


\section{3 $E_{n}$ value}

As a final evaluation of comparability of results of measurement methods $\mathrm{A}$ and $\mathrm{B}, E_{n}$ values were calculated for values for factor of adhesion $f_{R}$. Adhesion factors of were calculated using Simpson's equation. $E_{n}$ values for measured samples and values from which they were calculated are listed in Table 5.

\begin{tabular}{|c|c|c|c|c|c|c|c|c|}
\hline Sample & \multicolumn{2}{|c|}{10} & \multicolumn{2}{c|}{16} & \multicolumn{2}{c|}{28} & \multicolumn{2}{c|}{ 16-A } \\
\hline $\begin{array}{r}\text { Nethod } \\
\text { Chak }\end{array}$ & $\mathbf{A}$ & $\mathbf{B}$ & $\mathbf{A}$ & $\mathbf{B}$ & $\mathbf{A}$ & $\mathbf{B}$ & $\mathbf{A}$ & B \\
\hline$f_{R 1}$ & 0.0938 & 0.0839 & 0.0710 & 0.0671 & 0.0738 & 0.0703 & 0.0750 & 0.0829 \\
\hline$f_{R 2}$ & 0.0925 & 0.0840 & 0.0713 & 0.0647 & 0.0742 & 0.0696 & 0.0760 & 0.0820 \\
\hline $\bar{f}_{R}$ & 0.0932 & 0.0839 & 0.0711 & 0.0659 & 0.0740 & 0.0699 & 0.0755 & 0.0825 \\
\hline$U$ & 0.0052 & 0.0097 & 0.0052 & 0.0097 & 0.0052 & 0.0097 & 0.0052 & 0.0097 \\
\hline$E_{n}$ & 0.84 & 0.48 & 0.37 & \multicolumn{3}{c|}{0.63} \\
\hline
\end{tabular}

Tab. 5. $E_{n}$ values

Measurement results obtained using both methods are comparable. $E_{n}$ values of all samples are less than one, with declared measurement uncertainty of measurements obtained by the optical measuring device that was $U_{A}=$ $0.0052 \mathrm{~mm}, k=2, P=95 \%$ and the measurement uncertainty of measurements obtained by conventional hand-held measuring instruments that equals $U_{B}=$ $0.0097 \mathrm{~mm}, k=2, P=95 \%$.

\section{CONCLUSION}

This study theoretically and practically covered part of issues related to the measurement of geometric features of reinforcing steel, which are needed to determine the factors of adhesion between concrete and reinforcement.

Knowing the value of factors of adhesion, or the relative rib area is essential in making decisions about the steel that will be used as reinforcement. The importance of this information is indisputable if we note that reinforced concrete is the most common material in civil engineering today.

All the requirements to the reinforcing steel of concrete are laid down in standard ISO 1130:2008 and the methods needed to test these specific requirements in ISO 15630-1:2002. In accordance with the specified standards in the experimental part of this study, measurements were carried out to calculate the characteristics needed by the factors of adhesion by Simpson's equation $\left(a_{\mathrm{m}}, a_{1 / 4}, a_{3 / 4}, c, e\right)$. Measurements of these characteristics were made using two methods, for the purpose of comparison and to determine reliability of measurement with conventional hand-held measuring instruments in relation to measurement with the optical measuring device RE 302/202.
Results obtained by use of both methods were statistically analyzed in accordance with the requirements of standard ISO 5725-1984(E). Based on the result of the adhesion factor and calculated measurement uncertainty the $E_{n}$ values were determined for each sample.

By analyzing the results obtained with conventional hand-held measuring instruments and using the optical measuring device RM 302/202 it can be concluded:

- that the precision of measurements obtained in conditions of repeatability for the conventional handheld measuring instruments statistically less significant then precision of those measured with optical mesaurement device RM 302/202 in the case of measuring the characteristics $a_{\mathrm{m}}, c$ and $e$, while for the characteristics $a_{1 / 4}$ and $a_{3 / 4}$ we can not claim that. Noted is statistically confirmed in table 3 . in chapter 5,1 ;

- that the precision of mesaurement in general or precision of reproduced measurements with conventional hand-held measuring instruments are not statistically less significant than precision of mesaurment obtained with optical mesaurment device RM 302/202, for each cahracteristic. Noted is confirmed in table 4. in chapter 5.2.;

- that the measurement results obtained using one and other method are comparable, that was concluded on the basis of $E_{n}$ values calculated for the factor of adhesion $f_{R}$ with the declared measurement uncertainty of measurements obtained by optical measuring device RM 302/202 $U_{A}=0.0052 \mathrm{~mm}, k=$ $2, P=95 \%$ and the measurement uncertainty of measurement achieved using conventional hand-held measuring instruments $U_{B}=0.0097 \mathrm{~mm}, k=2, P=$ $95 \%$. Noted is confirmed in table 5. in chapter 5.3. of this paper.

\section{REFERENCES}

[1] Gukov I. (2009). Concrete Structures 1 - Lectures University of Zagreb - Faculty of Civil Engineering, Available from: http://www.scribd.com/doc/88684203/Predavanja-Beton-1-IgorGukov Accessed: 2012-04-01

[2] Radić J. et al. (2006). Concrete Structures - Handbook, Croatia University Press, University of Zagreb - Faculty of Civil Engineering, Available from: http://www.scribd.com/doc/35289528/Jure-Radi-Betonskekonstrukcije Accessed: 2012-04-11

[3] HRN 1130-2:2008, Steel for the reinforcement of concreteWeldable reinforcing steel - 2 Part: Technical delivery conditions for steel grade B

[4] EN 10080:2005, Steel for the reinforcement of concrete Weldable reinforcing steel - General

[5] EN ISO 15630-1:2002, Steel for the reinforcement and prestressing of concrete — Test methods - Part 1:Reinforcing bars, wire rod and wire

[6] ISO 5725-1:1994, Accuracy (trueness and precision) of measurement methods and result

[7] Pavlić I (1985). Statistical theory and application / Ivo Pavlic, Textbooks, University of Zagreb - Manualia Universitatis Studiorum Zagrebiensis, Technical Books 